\title{
Apuntes y debates en torno al Nuevo Modelo de Reconocimiento y Medición de Grupos e Investigadores en Colombia
}

\author{
Julio César Ossa \\ Fundación Universitaria de Popayán (Colombia) \\ Jean Nikola Cudina \\ Bibliometrics Research Lab (Colombia) \\ Claudia Burbano \\ Fundación Universitaria de Popayán (Colombia)
}

This work is licensed under CC BY-NC-ND

\section{Introducción}

El 25 de febrero del año 2021, el recientemente constituido Ministerio de Ciencia Ministerio de Ciencia, Tecnología e Innovación en Colombia abrió la “Convocatoria nacional para el reconocimiento y medición de grupos de investigación, desarrollo tecnológico o de innovación y para el reconocimiento de investigadores del Sistema Nacional de Ciencia, Tecnología e Innovación - SNCTI 2021” (MINCIENCIAS, 2021).

Dicha convocatoria, identificada con el Número 894, tiene como objetivo "Contar con información actualizada de los Grupos de Investigación, Desarrollo Tecnológico o de Innovación y los investigadores del país, sus actividades y los resultados logrados para generar conocimiento sobre las capacidades, fortalezas, debilidades y potencialidades de quienes integran el Sistema Nacional de Ciencia Tecnología e Innovación (SNCTI) ${ }^{1}$.

Para esta oportunidad el modelo conceptual con el que se cobija los términos de referencia de la presente convocatoria trajo consigo cambios sustanciales para el reconocimiento de Grupos e Investigadores en el SNCTI. Los apuntes que aquí se exponen tienen como propósito establecer una revisión a los ajustes que se realizaron al modelo de medición nacional. Se reflexiona sobre las implicaciones que traen consigo estos ajustes para

\footnotetext{
${ }^{1}$ Ver el siguiente link https://minciencias.gov.co/convocatorias/fortalecimiento-capacidades-para-lageneracion-conocimiento/convocatoria-nacional-para) para mayor detalle del proceso.
} 
efectos de la presente convocatoria y a su turno, se debaten algunos desafíos que versan sobre el fomento de una cultura de investigación y producción de conocimiento en el país.

\section{Cambios en los criterios de clasificación de grupos de investigación}

En enero de 2020 se conformaron diversas mesas y grupos de trabajo para discutir el modelo conceptual de reconocimiento de grupos e investigadores del Colombia (Ministerio de Ciencia y Tecnología, 2021). Estas comisiones se conformaron con el propósito de revisar los indicadores de calidad y fomentar prácticas de investigación con patrones y estándares internacionales.

Cada uno de estos grupos de trabajo incidieron en los ajustes y propuestas al modelo de medición en tres ejes fundamentales, a saber:

\section{La Unidad de Cienciometría de la Dirección de Generación de Conocimiento,} ajustes y cambios alrededor de los criterios de clasificación de grupos de investigación.

\section{La Mesa Técnica de expertos encargados de revisar las definiciones para libros y} capítulos de libros, cambios y aportes frente a los requerimientos de existencia, calidad, mecanismos de validación y definición de nuevas tipologías de publicaciones, de acuerdo con buenas prácticas internacionales de gestión editorial.

La Dirección de Capacidades y Divulgación de la CTeI, actualización de los productos resultados de procesos y actividades de Apropiación Social del Conocimiento y Divulgación Pública de la Ciencia.

Tabla 1.

Ajustes generales a criterios de clasificación de los Grupos de Investigación colombianos (año 2021)

\begin{tabular}{cc}
\hline Convocatoria de evaluación 2018 & Convocatoria de evaluación 2021 \\
\hline Contar con un indicador de Grupo & Contar con un indicador de Grupo \\
\hline $\begin{array}{c}\text { A pontar con un indicador de productos TOP y/o Tipo } \\
\text { social del conocimiento }\end{array}$ & $\begin{array}{c}\text { Contar con un indicador de productos TOP y/o Tipo } \\
\text { A }\end{array}$ \\
\hline $\begin{array}{c}\text { Contar con un indicador de productos apropiación } \\
\text { Contar con indicadores de productos derivados de } \\
\text { actividades de formación de Talento Humano }\end{array}$ & $\begin{array}{c}\text { Tener un indicador de Productos de Apropiación } \\
\text { Social del Conocimiento y Divulgación Pública de } \\
\text { la Ciencia* }\end{array}$ \\
\hline Contar con un indicador de cohesión & $\begin{array}{c}\text { Tener un Indicador de Productos de Actividades } \\
\text { Relanadas con la Formación de Recurso } \\
\text { Humano }\end{array}$ \\
\hline Contar al menos con dos años de existencia & $\begin{array}{c}\text { Contar con un indicador de cohesión } \\
\text { Contar con un indicador de Estabilidad de la } \\
\text { producción** }\end{array}$ \\
\hline $\begin{array}{c}\text { Contar con un indicador de permanencia de } \\
\text { investigadores** }\end{array}$ \\
\cline { 2 - 2 }
\end{tabular}


Para efectos de la convocatoria de medición y reconocimientos de Grupos 2021 se establecieron modificaciones en lo que respecta a los criterios de existencia de productos Tipo A y-específicamente con la tipología de Libros y Capítulos de libros- resultados de investigación y se hicieron ajustes al indicador de apropiación social de conocimiento. A demás de ello, se incluyeron tres indicadores de evaluación que no se contemplaban en pasadas convocatorias. A continuación, se presentan estos cambios.

\section{Libros y capítulos libros resultados de investigación}

El nuevo modelo conceptual para la medición de grupos. establece una nueva forma de concebir los libros y capítulos libros resultados de investigación. Los requerimientos de calidad, que se deben cumplir para vincular libro y capítulos de libros, hacen que cambie por completo la manera como se venían registrando los libros de investigación en pasadas convocatorias (Ver la Tabla 2).

Tabla 2 .

Ajustes generales a criterios de clasificación de Libros y capítulos libros resultados de investigación

\begin{tabular}{|c|c|c|}
\hline \multirow{2}{*}{ Tipología } & \multicolumn{2}{|c|}{ Indicadores de calidad } \\
\hline & Convocatoria 2018 & Convocatoria 2021 \\
\hline \multirow[b]{2}{*}{ Libro A1 } & \multirow{2}{*}{$\begin{array}{c}\text { Libro B que ha sido citado en } \\
\text { Revista A1, A2, B, C, o en libros } \\
\text { categoría B }\end{array}$} & $\begin{array}{l}\text { Libro con uno o varios premios o } \\
\text { menciones especiales }\end{array}$ \\
\hline & & $\begin{array}{l}\text { Libro resultado de investigación } \\
\text { ubicado en el primer cuartil de su } \\
\text { gran área de conocimiento }\end{array}$ \\
\hline Libro A & $\begin{array}{c}\text { Libro B que ha sido citado en } \\
\text { Revista D }\end{array}$ & $\begin{array}{l}\text { Libro resultado de investigación } \\
\text { ubicado en el segundo cuartil de su } \\
\text { gran área de conocimiento }\end{array}$ \\
\hline \multirow[b]{2}{*}{ Libro B } & $\begin{array}{l}\text { Libro que hace parte del Book } \\
\text { Citation Index (Web of Science- } \\
\text { Clarivate) o Scopus. }\end{array}$ & \multirow{2}{*}{$\begin{array}{l}\text { Libro resultado de investigación } \\
\text { ubicado en el tercer cuartil de su } \\
\text { gran área de conocimiento }\end{array}$} \\
\hline & $\begin{array}{l}\text { Libro que cumple con requisitos del } \\
\text { Anexo } 5 \text { y fue avalado } \\
\text { Institucinalmente el GrupLac y el } \\
\qquad v L a\end{array}$ & \\
\hline Capítulo A1 & $\begin{array}{c}\text { Capítulo de un libro resultado de } \\
\text { investigación B que ha sido citado } \\
\text { en Revista A1, A2, B o C o en } \\
\text { Libros categoría B }\end{array}$ & $\begin{array}{l}\text { Capítulo resultado de investigación } \\
\text { ubicado en el primer cuartil de su } \\
\text { gran área de conocimiento }\end{array}$ \\
\hline Capítulo A & $\begin{array}{l}\text { Capítulo que ha sido citado en } \\
\text { revista categoría D }\end{array}$ & $\begin{array}{l}\text { Capítulo resultado de investigación } \\
\text { ubicado en el segundo cuartil de su } \\
\text { gran área de conocimiento }\end{array}$ \\
\hline
\end{tabular}




\begin{tabular}{|c|c|c|}
\hline \multirow{4}{*}{ Capítulo B } & $\begin{array}{c}\text { Capítulo que hace parte del Book } \\
\text { Citation Index (Web of Science- } \\
\text { Clarivate) o Scopus }\end{array}$ & $\begin{array}{c}\text { Capítulo resultado de investigación } \\
\text { ubicado en el tercer cuartil de su } \\
\text { gran área de conocimiento }\end{array}$ \\
\cline { 2 - 3 } & $\begin{array}{c}\text { Capítulo que cumple con requisitos } \\
\text { del Anexo 5 y fue avalado } \\
\text { Institucinalmente el GrupLac y el } \\
\text { CvLac }\end{array}$ & \\
\hline
\end{tabular}

Esta tabla arroja un balance sobre los cambiones en este indicador de producción:

- En primer lugar que los libros y capítulos de libro resultados de investigación NO a través de la misma casa editorial de los Grupos de Investigación.

- Que un mismo producto (Libro) y capítulos de libro sumen al indicador de grupo y categorización de Investigador. Esto queda diferenciado como podrá observarse en el modelo y en las sección de preguntas que se encuentran más delante...

- Ya un libro no será A1 sólo por una cita en Revista indexada, ahora requiere quedar en el primer cuartir de su gran área de conocimiento y dichas citas no deben ser autocitas. Valga la pena decir que las citas no provengan de la misma filiación de los autores (o del autor) de la obra nos parece un exceso de la norma.

\section{Cambios en los indicadores de apropiación Social del Conocimiento}

El principal cambio realizado a este indicador se desprende a partir de los procesos de actualización de los productos resultados de procesos y actividades de Apropiación Social del Conocimiento y Divulgación Pública de la Ciencia, sus definiciones, requerimientos de existencia y calidad, respondiendo a los principios rectores de la Política pública de Apropiación Social del Conocimiento y Divulgación Pública de la Ciencia. Con estas actualizaciones se busca, integrar de manera acertada y pertinente el enfoque de Apropiación Social del Conocimiento y Divulgación Pública de la Ciencia a las actividades de investigación, sus resultados, impactos sociales, los equipos que participaron y los contextos en los cuales se desarrollaron; con base en los mencionados principios rectores ${ }^{2}$ (MINCIENCIAS, 2020).

Un aspecto importante para tener en cuenta en función de este cambio tiene que ver que para efectos de los términos de referencia de la presente convocatoria ya no se van a considerar productos derivados de indicadores de apropiación social del conocimiento tipo

\footnotetext{
${ }^{2}$ Referencia al documento de la política publica de apropiación social de conocimiento
} 
TOP y A como sí se había estipulado en la convocatoria anterior del $2018^{3}$. Los productos de Apropiación Social tuvieron un ajuste como indicador propio en la ecuación del modelo. A demás de esto, se incluyeron 4 productos y actualización de 4 productos adicionales en la tipología de Apropiación Social del Conocimiento ${ }^{4}$ a saber: Procesos de Apropiación Social del Conocimiento, Circulación de conocimiento especializado, Divulgación Publica de la CTeI y Bibliográfica.

\section{Inclusión de los indicadores de Permanencia, Trayectoria y de Estabilidad}

En los términos de referencia de la convocatoria 894 los indicadores de permanencia, trayectoria y de estabilidad se incluyen como una novedad en el proceso de medición y reconocimiento de grupos de Investigación e Investigadores ante el SNCTI. El Ministerio de Ciencia, Tecnología e Innovación ha incluido estos indicadores en el modelo conceptual con el propósito de brindar una información concerniente a la trayectoria en el tiempo de los grupos de investigación del país y a su turno, exponer indicadores de fortaleza y cohesión de a través de la permanencia de los investigadores en el grupo y su productividad.

Para tal fin, el modelo conceptual precisa en un indicador de trayectoria y dos indicadores de estabilidad, a saber: Permanencia de los Investigadores y frecuencia de publicación de resultados de nuevo conocimiento. En lo que respecta, al indicador de trayectoria se tienen en cuenta el comportamiento histórico de los grupos de investigación en cada una de las últimas cinco (5) convocatorias de reconocimiento y medición de grupos e investigadores en el país. En lo que respecta al indicador de permanencia de investigadores es importante mencionar que se tomará en cuenta para efectos de la presente convocatoria únicamente a investigadores reconocidos por el sistema de investigadores del Ministerio, más las personas que figuran con formación doctoral en los grupos de investigación. El indicador de estabilidad, de producción se calcula a partir de los productos de nuevo conocimiento, sin ninguna distinción particular 5 .

\section{Algunos apuntes al modelo de medición}

\footnotetext{
${ }^{3}$ Ver tipología de productos tipo TOP y A de modelo conceptual para medición de grupos 2018

${ }^{4}$ Para más detalle revisar Anexo 1. Modelo de medición de grupos Apartado "Productos resultados de actividades de apropiación social y divulgación publica

${ }^{5}$ El calculo de estos indicadores se tomo a partir de relevamiento de información de los grupos en pasadas convocatoria a través de un modelo estadístico. Revisar Anexo 4 "Modelo Estadístico para la trayectoria de los grupos de Investigación"
} 
Para esta Convocatoria, se define como "ventana de observación el período comprendido entre el 1 de enero de 2016 al 31 de diciembre de 2020" (MINCIENCIAS, 2021). Como se puede observar esta ventana incluye el año 2020, año en el cual por efectos de COVID-19 el Gobierno Nacional expidió el Decreto 417 el 17 de marzo del 2020 por el cual se declara se declara un Estado de Emergencia Económica, Social y Ecológica en todo el territorio Nacional (Función Pública, 2020). Tiempo en el cual buena parte de las IES suspendieron sus actividades educativas, y con ellas su función sustantiva de investigación.

Pese a que la ventana de observación incluye este periodo de pandemia, llama la atención que en su primera edición del modelo de medición (única hasta el momento de esta publicación) no se mencione esta contingencia. De hecho, una simple inspección al documento permite evidenciar que la palabra "Covid" no aparece en el documento. Frente a ello surge una duda, una crisis social, económica y sanitaria de esta naturaleza ¿No afecta las dinámicas de investigación en sus diferentes dimensiones? (salidas de campo, ...; ...; entre otros)?

Lo anterior llama nuestra atención -como ya lo hemos dicho- en particular porque en Colombia se viene insistiendo en la necesidad de una investigación con impacto social ¿A caso los indicadores de Apropiación Social del Conocimiento no se vieron afectados durante este año de pandemia? Esto queremos resaltarlo porque esta tipología de productor fueron unas de aquellas que presentaron cambios en sus indicadores (valor global y ponderación en la ecuación) en el Nuevo Modelo de Medición.

Por otro lado, quisiéramos plantear una reflexión en torno a una cuestión con relación a las normativas, leyes y regulaciones de nuestro Gobierno en Colombia; esto en el sentido del alcance de cada una de ellas cuando es promulgada. Constitucionalmente, en nuestro País, ninguna Ley puede tener un alcance retroactivo, salvo los casos que se considere que el alcance de la Ley o norma debe ser retrospectivo.

Parece que los Grupos de Investigación son ajenos a este principio fundamental. Cada vez que un Modelo de Medición entra a regir su alcance es para actividades de investigación realizadas durante una ventana de observación del pasado. La entrada en vigor de cada modelo, según sean los ajustes y cambios realizados a los criterios de medición, puede afectar incluso aquellos productos que ya habían sido evaluados en convocatorias anteriores. Como 
se observa en el actual Modelo de Medición no hay ninguna duda en el alcance retrospectivo de su disposición normativa.

\section{Preguntas e interrogantes al modelo de medición}

La puesta en marcha de los términos de referencia de la presente convocatoria al modelo de medición y reconocimiento de grupos e investigadores del país suscita interrogantes ante los cambios y modificaciones establecidas. Ante ello, se formularon una serie de preguntas oficiales al Ministerio de Ciencia, Tecnología e Innovación en Colombia en función de los tres ejes más representativos donde acontecieron los ajustes al modelo, a saber: la nueva tipología de libros y capítulos de libros, Indicadores de apropiación Social del Conocimiento e indicadores de Permanencia, Trayectoria y de Estabilidad-

\section{Consulta respecto a la tipología de libros y capítulos de libros resultados de investigación}

\section{Pregunta Autores (P.A)}

¿Qué pasará con los libros y capítulos de Libros que fueron registrados como resultados de investigación en pasadas convocatorias y que tienen vigencia en la ventana de observación de la presente convocatoria pero que no se ajustan con los criterios de calidad del nuevo modelo?

\section{Respuesta MINCIENCIAS (R.M)}

Cada proceso de reconocimiento y medición es único y diferente uno de otro, por esta razón los productos deben cumplir de manera estricta la definición y requerimientos de existencia y calidad relacionados en el documento conceptual de la Convocatoria (Bogotá D.C., 04-03-2021).

P. A: De no ser reconocidos los libros y capítulos como resultados de investigación para efectos de la presente convocatoria muchos grupos de investigación e investigadores reducirían sus indicadores de Productos Tipo TOP y A sustentado en libros y capítulos de libro (Categorizados como A1, A, B en pasadas convocatorias). Con el ajuste a la tipología del libro y capítulo del nuevo modelo se ¿anularía o no reconocería el histórico de productos tipo top y a de un grupo e investigador? ¿Qué implicaciones trae esto para la categorización de grupos e investigadores?

R. M: Como se mencionó, cada proceso de reconocimiento y medición es diferente uno del otro, y relacionan diferentes métricas de medición de los productos resultados de investigación. No es posible determinar por ahora, si muchos grupos de investigación, como dice la comunicación, reducirían sus indicadores, teniendo en cuenta que no son los únicos que se tienen en cuenta para otorgar una categoría (Bogotá D.C., 04-03-2021). 
P. A: Los capítulos que componen una obra deben derivarse de ¿Un único proyecto de investigación? $\mathrm{O}$ ¿Puede ser una investigación macro y otro(s) proyecto(s) financiados y apoyados por Universidades diferentes a la institución que avala la obra?

R. M: Los capítulos de libro de investigación son derivados de procesos de investigación. En el apartado 2.2.1.4 (capítulo 2) de los términos de referencia de la Convocatoria, se encuentra relacionada la definición y requisitos de los capítulos en libro resultado de investigación. Se validarán los capítulos que hacen parte de libros compilatorios, cuando estos cumplan de manera estricta el concepto, los requerimientos de existencia y de calidad definidos para "Capítulos Resultado de Investigación" que se relacionan en el documento conceptual. No se contarán los libros de compilaciones como unidades adicionales a los capítulos registrados (Bogotá D.C., 04-03-2021).

Todos los capítulos de un libro resultado de investigación en coautoría, deben ser escritos por los mismos autores. De lo contrario se considerará esta publicación como un libro compilatorio y se evaluarán los capítulos como unidades separadas, de acuerdo con lo establecido en el documento conceptual (Bogotá D.C., 04-03-2021).

P. A: Los REQUERIMIENTOS DE CALIDAD para un Libro LIB_A1 deben cumplirse AMBOS, es decir el libro debe tener uno o varios premios o menciones especiales y a su vez debe estar ubicado en el primer cuartil superior (de su gran área de conocimiento).

R. M: No. Deben cumplir con alguna de las dos condiciones (Bogotá D.C., 04-032021).

P.A: ¿Cuál será la metodología para el calculo de los cuartiles, sabiendo que Google Scholar, Scopus y Web of Science son fuentes de información bastante heterogéneas?

R. M: El Ministerio cuenta con un software especializado para la identificación de las citaciones y el cálculo de los cuartiles (Bogotá D.C., 04-03-2021).

\section{Consulta respecto al eje de apropiación Social del Conocimiento y Divulgación Pública de} la Ciencia

P. A: El modelo conceptual de grupos trae un ajuste al eje de "apropiación social del conocimiento" este ajuste trae consigo cambios a la categorización de productos en esta línea. En convocatorias pasadas se contemplaban productos derivados de apropiación social de conocimiento como productos tipo TOP y A. Para efectos de la presente convocatoria no se contempla ningún producto de apropiación social como un producto tipo TOP y A. ¿Que consecuencias trae esto para la clasificación de los grupos e investigadores? Además de pensar un enfoque "de apropiación social de conocimiento y divulgación publica de la ciencia") "Qué relación trae este ajuste con el documento oficial de los "Lineamientos para una política Nacional de apropiación Social del Conocimiento. ¿Ciencia, Tecnología e Innovación de los ciudadanos para los ciudadanos? 
R.M: Los productos de Apropiación Social, han tenido un indicador propio. Los productos Tipo Top o Tipo A, son solo para productos de la más alta trayectoria y excelencia en nuevo conocimiento y desarrollo tecnológico e innovación. Los productos relacionados en el documento responden a los lineamientos y dimensiones contempladas en la Política de Apropiación Social del Conocimiento.

\section{Consulta respecto al eje de Indicadores de Permanencia, Trayectoria y de Estabilidad}

P.A: Si bien es cierto que los indicadores de trayectoria buscan brindar información del recorrido de los grupos de investigación a través del tiempo, específicamente, en función de las últimas cinco (5) convocatorias de medición de grupos ¿Qué implicaciones trae el cálculo de este indicador para la categorización de los grupos de investigación? Esto si consideramos que en el país existen grupos que no se han presentado necesariamente a todas las convocatorias de reconocimiento y medición. A demás de ello ¿Qué implicación trae este indicador para aquellos grupos de investigación jóvenes, que consolidan al menos dos años de existencia y cumplen con el criterio de reconocimiento?

P.A: En lo que respecta al indicador de permanencia de investigadores, el modelo conceptual establece que para dicho calculo se tomara como referencia a investigadores reconocidos por el sistema de investigadores del Ministerio, más las personas que figuran con formación doctoral en los grupos de investigación ¿Este calculo se basa en el relevamiento de información de pasadas convocatorias? $\mathrm{O}$ el calculo ¿contempla la emergencia de investigadores vigentes, reconocidos y categorizados en el nuevo modelo de medición?

P.A: En caso dado que un investigador sea categorizado o no de acuerdo con los criterios de evaluación de la presente convocatoria ¿Sería objeto de medición en el indicador de permanencia? ¿Qué implicaciones tendría este indicador para el reconocimiento de investigadores en el país? ¿Estos indicadores estarían develando el cumplimiento de criterios para alcanzar la categorización de investigadores en futuras convocatorias?

P.A: Qué pasaría con el cálculo del indicador de permanencia de un investigador que por condiciones contractuales ha tenido que cambiar de grupos de investigación ¿Se vería afectado el cálculo de este índice? ¿Esto también afectaría el indicador de estabilidad del investigador?

\section{Algunas consideraciones al fomento de la investigación y producción de conocimiento en Colombia}

El nuevo modelo de medición y reconocimiento de grupos de investigación trae consigo preguntas e interrogantes en función de un proceso de asimilación de lo que podríamos estar considerando la ruta y horizonte del fomento de la investigación y 
producción académica en el país en esta década que comienza. Esperamos que esta nota contribuya con los debates y reflexiones que nos permita establecer un dialogo conjunto con la comunidad académica de lo que sería la consolidación de este proyecto de fomento en un futuro mediano.

En función de los tres ejes expuestos que conllevaron a modificaciones de fondo en la manera como habitualmente estábamos acostumbrados a conocer y prepararnos en los procesos de evaluación de grupos e investigadores, podemos develar algunos aspectos para dejar en consideración a través de un debate abierto en función de las dinámicas de investigación en Colombia.

En primer lugar, el modelo conceptual permite develar el fomento de unas dinámicas de investigación que se proyectan hacia el crecimiento y fortalecimiento de indicadores de internacionalización de la ciencia colombiana. La visibilización de la producción académica local, de la investigación nacional en los escenarios de la comunidad académica internacional es un aspecto que se viene posicionando con fuerza en el último quinquenio. Esto no es un aspecto nuevo. Vale la pena recordar, en su momento los ajustes al modelo de medición de revistas científicas nacionales (Caballero-Uribe \& Viloria-Doria, 2018; Ossa \& Cudina, 2016).

Este aspecto constituye un punto importante para ir forjando una cultura científica nacional de producción científica de cara a su impacto e internacionalización, pero no constituye un determinante. Esto si pensamos que existen diversas instituciones y practicas investigativas en el país que no se proyectan hacia estas lógicas de producción de alto impacto.

Es por ello por lo que el desarrollo de una nueva cultura de investigación que promueva esta lógica de alto impacto y practicas con estándares internacionales a través de una política nacional de fomento de la ciencia debe considerar garantizar el avance hacia este horizonte por parte de la comunidad académica del país con el acompañamiento y la infraestructura necesaria que permita hacer un seguimiento optimo por parte del Ministerio de Ciencia Tecnología e Innovación hacia este fin.

Un terreno importante para avanzar hacia este propósito podríamos encontrarlo con el ensamble de promover dinámicas de investigación a través del eje de apropiación Social del Conocimiento y Divulgación Pública de la Ciencia por medio de unos lineamientos 
nacionales de una política pública de apropiación social del conocimiento (MINCIENCIAS, 2020). Esto representa un segundo aspecto que podemos develar del modelo medición y reconocimiento de grupos e investigadores ante el SNCTeI.

Esta propuesta claramente se constituye en un eje importante para estrechar las brechas que se generan entre la academia y la comunidad. Establecer el acercamiento y poder conocer el impacto tangible de las dinámicas de investigación en la sociedad es uno de los desafíos que depara el fomento de la ciencia colombiana en esta nueva década. En todo caso, se requiere de mecanismos y rutas de acceso que nos permitan avanzar hacia esta vía.

\section{Referencias}

Caballero-Uribe, C. V., \& Viloria-Doria, J. C. (2018). Un análisis del impacto del nuevo modelo de clasificación de revistas científicas según Colciencias. Salud Uninorte, 34(1), 7-12.

Función Publica (2020). Decreto 417 de 2020. Estado de Emergencia Económica, Social y Ecológica en todo el territorio Nacional. República de Colombia.

MINCIENCIAS. (2020). Lineamientos para una Política Nacional de Apropiación Social del Conocimiento Ciencia, Tecnología e Innovación de los ciudadanos para los ciudadanos. República de Colombia.

MINCIENCIAS. (2021). Convocatoria nacional para el reconocimiento y medición de grupos de investigación, desarrollo tecnológico o de innovación y para el reconocimiento de investigadores del Sistema Nacional de Ciencia, Tecnología e Innovación - SNCTI 2021. República de Colombia.

Ossa, J. C., \& Cudina, J. N. (2016). Sesenta años de las revistas de psicología en Colombia. Revista Guillermo de Ockham, 14(2), 7-17. 